Esta revista forma parte del acervo de la Biblioteca Jurídica Virtual del Instituto de Investigaciones Jurídicas de la UNAM

\title{
Shelby County vs. Holder. Derecho al voto, minorías raciales y defensa del federalismo en los Estados Unidos*
}

\section{Víctor M. Colli Ek**}

\section{Sumario:}

\author{
I. Un problema complejo \\ II. Las enmiendas de la reconstrucción \\ III. Voting Rights Act y sus ratificaciones \\ IV. Antecedentes jurisprudenciales \\ V. La decisión y sus votos minoritarios \\ VI. Lecturas sobre su actualidad
}

* El presente estudio es un producto del proyecto de investigación: "La vigencia de la Constitución en la interpretación de la Suprema Corte de Justicia de la Nación como Tribunal Constitucional", 154998, financiado por el Conacyt, a través del fondo de Ciencia Básica, en el que el autor es responsable.

** Doctor en Derecho por la Universidad Panamericana; profesor-investigador en el Centro de Investigaciones Jurídicas de la Universidad Autónoma de Campeche.

D. R. (C) 2014. Universidad Nacional Autónoma de México-Instituto de Investigaciones Jurídicas. 


\section{Un problema complejo}

En el periodo octubre 2013, la Corte Roberts de los Estados Unidos, decidió un asunto importante y complejo dentro de la democracia norteamericana. Al estudiar la constitucionalidad de las secciones 4 y 5 de la Voting Rights Act (VRA) de 1965, la Corte no puede evitar pronunciarse sobre temas constitucionales complejos. La variedad de elementos que implicó, pasa por la historia de la lucha del reconocimiento de los derechos civiles - en especial el derecho de voto para los afroamericanos- y por tanto cómo se ha enfrentado este problema. Al igual, bajo este asunto, se discutió la afectación al diseño del Federalismo - fórmula consolidada por Estados Unidos en los tiempos modernos, en especial, el análisis de la legitimidad de la facultad del Congreso de la Unión, para imponer obligaciones a los estados-. Finalmente, pasa por una consecuencia clara de esta discusión del diseño federal, el respeto uniforme de la soberanía de los estados de la Unión.

En el asunto - Shelby County vs. Holder- el Chief Justice Roberts, redactando la decisión, se pronunciaría sobre una parte importante de la VRA, creada por el Congreso mediante los amplios poderes - sweeping powers - que derivaron de la Enmienda 15. Bajo esta ley, determinados estados de la Unión Americana estaban impedidos para modificar sus leyes electorales de manera unilateral y espontánea, porque necesitaban de una preautorización - pre-clearence- del gobierno federal, para poder hacer algún cambio. La decisión concluyó que este procedimiento de preautorización, implicaba una afectación al diseño federal y un trato desproporcionado a la soberanía de algunos estados.

Igualmente se ventiló si efectivamente el Congreso tenía amplios poderes para hacer una determinación de esta naturaleza. Y finalmente, se resolvió que el modo en que se designan qué estados deben cumplir con este requisito, no obedece a las circunstancias actuales, por lo que su existencia, no tiene justificación alguna.

Como veremos la discusión que enmarca el caso Shelby, proviene de décadas atrás, tiene sus raíces inclusive en las consecuencias constitucionales de la guerra civil. Por este motivo, el marco bajo el que haremos la exposición abarca los siguientes elementos. Primero, exposición de las Enmiendas de la "Reconstrucción”, pues la VRA analizada, se de- 
sarrollará dentro de la dinámica iniciada con estas enmiendas, la lucha por los derechos civiles de las enmiendas 13 y 14 y el reconocimiento del derecho de voto señalado en la enmienda 15. Segundo, exposición de la VRA y sus posteriores ratificaciones, hasta llegar al diseño analizado por la Corte. Tercero, los antecedentes jurisprudenciales del caso Shelby. Cuarto, el análisis del caso a través de la decisión mayoritaria del Chief Justices John Roberts, el voto concurrente del juez Clarence Tomas y el voto disidente de la jueza Ruth Bader Ginsburg. Finalmente, haremos una sumaria mención de algunas implicaciones del tema a la realidad actual.

\section{Las enmiendas de la reconstrucción}

"The story of black ballots begins with black bullets" dice el profesor Akhil Reed Amar, la Guerra Civil fue producto de la confrontación entre los estados del norte y los del sur en los Estados Unidos, marcada por la victoria presidencial de Lincoln en noviembre de 1860 - defensor de la igualdad y la abolición de la esclavitud-, en donde originalmente seis estados se separarían para formar a los Estados Confederados de América, con Texas iniciando igualmente su separación del resto de la nación.

Ganada la guerra por los estados antiesclavistas del norte, algo quedaba claro, ningún estado ex confederado debía ser aceptado de nuevo en la Unión, a menos que se adhiriera y siguiera haciéndolo, a las reglas de la Constitución, esto generaría "the First Reconstruction Act", donde el Congreso indicaría lo que los esos estados debían hacer, para que pudieren ser admitidos:

First, each ex-Confederate state should adopt a new state constitution via an electoral process enfranchising virtually all adult male residents, regardless of race. Second, each new state constitution should guarantee a right to vote in ordinary elections to this same broad swath of adult male residents. Third — and most important for our purposes - the new

1 Reed Amar, Akhill, America's Constitution. A biography, USA, Random House, 2006, p. 396. 
Esta revista forma parte del acervo de la Biblioteca Jurídica Virtual del Instituto de Investigaciones Jurídicas de la UNAM

governments elected under the new state constitutions should ratify the Fourtheenth Amendment, wich Congress had proposed in June $1866 .{ }^{2}$

La evolución del reconocimiento jurídico del derecho de voto para los afroamericanos se daría dentro de un proceso de retroalimentación. Primero, la participación e influencia en la victoria de los esclavos negros, en la guerra civil, será determinante para este proceso, y para la configuración de las enmiendas de la reconstrucción. En los años 1866-1868, los estados del norte impondrían el sufragio de los negros a los estados confederados, mientras se excluían ellos. En 1869-1870, la unión entre los votantes negros del sur y los legisladores negros al Congreso de la Unión, provocaría el cambio para el norte.

Marco importante para entender esta decisión, son las enmiendas de la reconstrucción 13, 14 y 15 a la Constitución de los Estados Unidos. Estas se dieron posteriormente a la proclamación del fin de la esclavitud, y en ellas se consolidaba el nuevo estatus de los ex esclavos.

Las enmiendas citadas - que vistas desde el presente parecen tener animación sistemática, aunque históricamente obedecieron a dinámicas propias e independientes, cada una con problemas que sortear y fines particulares que lograr - tienen como objetivo, otorgar igual protección ante la ley, ofrecer los mismos privilegios a todos los ciudadanos y conceder el derecho de voto.

La enmienda 13: "Neither slavery nor involuntary servitude", se daría en 1865, y su objetivo sería acabar con la esclavitud. La 14: "No State shall"3 - como diría Akhil Reed Amar, genera la incorporación del Bill of Rights de 1789 para los estados,$-{ }^{4}$ de 1866 y ratificada en 1868, buscaba hacer a todas las personas nacidas en América, ciudadanos iguales, protegiendo todos sus derechos fundamentales contra invasiones federales y estatales. La 15: "The rights...to vote", ${ }^{5}$ propuesta

2 Akhill Reed Amar, America's unwritten constitution, USA, Basic Books, 2012 p. 80.

3 No State shall make or enforce any law which shall abridge the privileges or immunities of citizens of the United States; nor shall any State deprive any person of life, liberty, or property, without due process of law; nor deny to any person within its jurisdiction the equal protection of the laws.

4 Akhill Reed Amar, America's Constitution, cit., p. 386.

5 . The right of citizens of the United States to vote shall not be denied or abridged by the United States or by any State on account of race, color, or previous condition of servitude. 2 . The Congress shall have power to enforce this article by appropriate legislation. 
Esta revista forma parte del acervo de la Biblioteca Jurídica Virtual del Instituto de Investigaciones Jurídicas de la UNAM

en 1869 y ratificada en 1870, garantizaría a los negros los derechos políticos de votar, ser votados y desempeñar cargos públicos. ${ }^{6}$

Estas modificaciones constitucionales, dejaron claro que la república norteamericana estaba siendo refundada, bajo los principios de una libre e igual ciudadanía, invitando a todos a participar en el proyecto democrático.

\title{
III. Voting Rights Act y sus ratificaciones
}

\begin{abstract}
Ahora, el análisis del caso Shelby, se dio específicamente sobre la VRA, contenidos que como veremos, habían sido controversiales desde su inicio.
\end{abstract}

Lo primero que debemos tomar en cuenta, es que la VRA, se encuentra dentro del marco de estas enmiendas de la Reconstrucción, y en especial, apoyando a la idea de la enmienda 15. Es la apuesta político-jurídica del Congreso, de mantener una defensa afirmativa de los derechos del voto para los afroamericanos en los Estados Unidos.

The Voting Rights Act of 1965 was enacted to address entrenched racial discrimination in voting, "an insidious and pervasive evil which had been perpetuated in certain parts of our country through unremitting and ingenious defiance of the Constitution"?

Dentro de diversas fuentes que generarán la aparición de la VRA, encontramos la campaña en Alabama, condado de Dallas a inicios de 1965, donde se denunció que la Civil Rights Act de 1964, había fallado en otorgar previsiones para asegurar que los afroamericanos tuvieran el derecho de voto. Igualmente encontramos como causa inmediata los hechos del "Bloody Sunday", el 7 de marzo del mismo año, que

${ }^{6}$ Una afirmación importante que encontramos en la decisión Shelby y que sostendrá el sentido del voto mayoritaria será: "The Fifteenth Amendment commands that the right to vote shall not be denied or abridged on account of race or color, and it gives Congress the power to enforce that command. The Amendment is not designed to punish for the past; its purpose is to ensure a better future". Shelby County v. Holder, 570 U.S. 2013 Opinion, p. 21. En adelante Shelby County (Op).

7 Shelby County v. Holder, 570 U.S. 2013 Syllabus, p. 1. En adelante Shelby County (Syll). 
Esta revista forma parte del acervo de la Biblioteca Jurídica Virtual del Instituto de Investigaciones Jurídicas de la UNAM

serían transmitidos por televisión, dejando clara la aún dura realidad de la lucha por los derechos civiles. ${ }^{8}$

Esta ley, fue firmada por el presidente Lyndon Johnson el 6 de agosto de 1965, poniendo en la práctica el derecho de voto de los afroamericanos, sustentada en las disposiciones de la enmienda 15.

This gave Federal government officials the power to take over the registration processs in any district where less than 50 per cent of African-Americans were on the electoral register, or where it appeared that local officials were discriminationg against African-Americans. It also requiered stated to get clearence from the Federal government before they introduced any new electoral regulations, to make suere that new devices did not replace the old ones. ${ }^{9}$

La ley estaba diseñada para expirar en cinco años, pero fue ratificada en varias ocasiones más: en 1970, por el presidente Nixon, extendida por cinco años; en 1975, por el presidente Gerald Ford, extendida 7 años; 1982, por el presidente Ronald Reagan, ratificada por 25 años, y, en 2006 por el presidente George W. Bush, por 25 años más.

Como veremos, el problema central, tanto histórico como analizado en el caso Shelby, es este filtro o pre-clearence del gobierno federal, impuesto a algunos estados que se encuentra dentro de la VRA. Este filtro está compuesto por dos partes: primero - contenido en la sección 4- la determinación de qué estados deben cumplir con él; segundo - contenido en la sección 5-, el procedimiento mismo a seguir. Dicho esto, es importante tomar en cuenta, que el primer filtro, o la regla para determinar qué estados debían requerir la preautorización por parte del poder federal, fue actualizado en cada ratificación, de acuerdo a las necesidades históricas presentes, pero en 2006 no lo fue, usándose entonces las razones generadas para modificaciones anteriores, en especial las de 1975. Esto se debe tener en cuenta, porque precisamente, será el argumento decisivo de la Corte en el caso. ${ }^{10}$

${ }^{8}$ Cfr. Wiliam Storey, Us Government and Politics, Edimburg University Press, UK, 2007, p. 39 y 40.

9 William Storey, op. cit., p. 137.

${ }^{10}$ John Paul Stevens, "The Court and the Right to Vote. A dissent", The New York Review of Books (Bending toward justices. The voting rights act and the transformation of American Democracy. Gary May) http://www.nybooks.com/articles/archives/2013/ aug/15/the-court-right-to-vote-dissent/?pagination=false. 
Esta revista forma parte del acervo de la Biblioteca Jurídica Virtual del Instituto de Investigaciones Jurídicas de la UNAM

En este sentido se expresaría el juez Roberts: “The statute's coverage formula is based on data that is now more than 35 years old, and there is considerable evidence that it fails to account for current political conditions". ${ }^{11}$

\section{Antecedentes jurisprudenciales}

La Corte de los Estados Unidos, ya se había referido a la VRA y el filtro en dos ocasiones importantes, previamente.

Primero. South Carolina vs. Katzenbach 383, U.S. 301, resuelto el 7 de marzo de 1966, por la Corte Warren, con una votación de 8-1. En esta decisión se analizó la versión original de la VRA de 1965, específicamente la prohibición hacia los estados de usar test o instrumento para negar el derecho de voto, otorgando a los poderes federales, poder para intervenir o investigar, irregularidades sobre este derecho, lo que nos refiere a las secciones 4 y 5 principalmente.

La Corte sostuvo la constitucionalidad de la ley, argumentando que la enmienda 15 otorgaba al Congreso full remedial powers, para prevenir la discriminación racial en el voto, calificando a la ley como una respuesta legítima al insidious and pervase evil que había negado el derecho de voto a los afroamericanos, desde la adopción de la enmienda 15 en $1870 . .^{12}$

Segundo. Northwest Austin Municipal Util. Dist. No. One v. Mukasey 557 U.S. $193,{ }^{13}$ resuelto el 22 de junio de 2009, ya por la Corte Roberts - lo que otorgará una línea argumentativa entre esta decisión y Shelby-, con una votación de 9. En este asunto, se analizarían varios temas: primero, si la municipalidad podía verse beneficiada por el procedimiento para excluirse del requisito de preautorización. Segundo, si la sección 5 de la VRA, que es el procedimiento de preautoriza-

${ }_{11}$ Northwest Austin Municipal Util. Dist. No. One v. Mukasey 557 U.S. 193. Opinion.

12 Cfr.http://www.oyez.org/cases/1960-1969/1965/1965_22_orig.

13 http://www.scotusblog.com/case-files/cases/northwest-austin-municipal-utility-dis trict-number-one-v-eric-holder-jr-attorney-general-et-al/. Igualmente, $c f r$. Moriarty, Christopher F., "The demise of the voting rights act?: A preview of Northwest Austin Municipal District number one v. Holder", Duke Journal of Constitutional Law and Public, Policy Sidebar, vol. 4. 2009, pp. 459-469. 
ción, era constitucional. Tercero, si la legitimación del Congreso para tomar la decisión de ratificar la VRA por otros 25 años, era justificada.

La Corte indicó que efectivamente la municipalidad podía solicitar ser excluida del requisito de preautorización, pero no se pronunció sobre la constitucionalidad y tampoco el problema político más importante que era la legitimidad del Congreso para ratificar, aunque $-\mathrm{y}$ esto debe resaltarse- sí presumió que la ley planteaba preocupaciones trascendentes sobre su constitucionalidad, determinando que la fórmula de cobertura de la sección 4 no había sido actualizada, esto es, las razones que llevan a determinar qué estados deben seguir siendo preautorizados, aunque históricamente justificadas, como en el caso Katzenbach, no encontraban cabida más en 2009. Con esto abría la puerta a una posterior revisión, cuestión que haría en Shelby.

\section{La decisión y sus votos minoritarios}

El asunto ${ }^{14}$ inició por una petición de la municipalidad de Shelby, dentro del Estado de Alabama, uno de los 9 estados a los que se le aplica la preautorización. ${ }^{15}$

En 2010, el municipio demandó al fiscal general ante una Corte distrital en Washington, solicitando la inconstitucionalidad de las secciones 4 y 5 de la VRA, al igual que una orden permanente contra su aplicación. La Corte Distrital no otorgó la petición, argumentando que las razones del Congreso para reautorizar en 2006, en ambas secciones era justificada.

Esta decisión fue recurrida ante la Corte de Apelaciones. Esta Corte considerando el material de evidencia presentado, determinó igualmente, que la validez de las secciones 4 y 5 estaban aún justificadas.

La evidencia que llevó a la conclusión de esta Corte, fue clasificada en seis categorías:

Attorney General objections to voting changes, Attorney General requests for more in formation regarding voting changes, successful $\$ 2$ suits

14 Shelby County v. Holder, 570 U.S. 2013.

15 Aplica a 9 estados: Alabama, Alaska, Arizona, Georgia, Louisiana, Mississippi, South Carolina, Texas and Virginia. 
Esta revista forma parte del acervo de la Biblioteca Jurídica Virtual del Instituto de Investigaciones Jurídicas de la UNAM
in covered jurisdictions, the dispatching of federal observers to monitor elections in covered jurisdictions, $\$ 5$ preclearance suits involving covered jurisdictions, and the deterrent effect of $\$ 5 .^{16}$

La ponderación de estos elementos, hizo a la Corte de Apelaciones sostener la constitucionalidad de ambas secciones. Esto llevó a la municipalidad de Shelby a solicitar la intervención de la Corte Suprema de los Estados Unidos, por medio de Writ of Certiorari, lo que nos lleva a la decisión.

El asunto sería escuchado por la Corte Suprema de Estados Unidos el 27 de febrero y resuelto el 25 de junio, ambos de $2013 .{ }^{17}$

La decisión, aunque estudió la constitucionalidad las secciones 4 y 5 de la VRA, sólo resolvió sobre la sección 4, la que comprende el criterio de "cobertura", donde se indica qué Estados o gobiernos locales, ${ }^{18}$ caen dentro del requisito de pre-clearence de la sección 5.

Sobre esta medida de la VRA —en específico la constitucionalidad de la sección 5, la Corte Suprema ya se había pronunciado en 2009, de manera parcial- lo que quiere decir que no quiso pronunciarse sobre "el gran tema" que era la constitucionalidad de la medida y con esto anteponerse a una decisión del Congreso: ${ }^{19}$

But the court, in an 8 to 1 decision, assiduously sidestepped the broader question of whether the provision was constitutional given the civil rights gains of the past 40 years, leaving that for another day. Instead, it ruled

16 Shelby County (Op), p. 8.

${ }_{17}$ Cfr. http://www.scotusblog.com/case-files/cases/shelby-county-v-holder/.

18 The law had applied to nine states - Alabama, Alaska, Arizona, Georgia, Louisiana, Mississippi, South Carolina, Texas and Virginia - and to scores of counties and municipalities in other states, including Brooklyn, Manhattan and the Bronx. Adam Liptak, "Supreme Court Invalidates Key Parts of the Voting Rights Act", The New York Times, 25 de junio de 2013. http://www.nytimes.com/2013/06/26/us/supreme-court-ruling. html?pagewanted $=$ alle_ $r=0$.

19 Parafraseando al presidente de la Corte de los EUA: “Chief Justice John G. Roberts Jr., writing for the majority, acknowledged that the court's approach stretched the statutory text, but he said the court should avoid deciding hard constitutional questions when it could. "We are now a very different nation" than the one that first passed the Voting Rights Act, he said. "Whether conditions continue to justify such legislation is a difficult constitutional question we do not answer today", Liptak, Adam, "Justices retain oversight by U.S. on voting", The New York Times, 22 de junio de 2009, http://www.nytimes. com/2009/06/23/us/23scotus.html. 
Esta revista forma parte del acervo de la Biblioteca Jurídica Virtual del Instituto de Investigaciones Jurídicas de la UNAM

only that the utility district was a "political subdivision" that could seek to "bail out" of federal oversight when changing its voting procedures. ${ }^{20}$

Un elemento importante de esta decisión, a nivel de interacción entre poderes constitucionales, como señala Robert G. McCloskey, es el empleo del dialogical model of interaction, entre la Corte y el Congreso:

Interestingly enough, the opinion included a great deal of language casting doubt on the constitutionality of requiring preclearence at all, and several analysts read it as an invitation to Congress to revisit the act and, in effect, bring it more in line with contemporary realities rather targeting the act by and large on a number of Southern stated for misconduct that occurred almost half a century ago. ${ }^{21}$

En ese sentido, la Corte —-dando lugar a la reacción del Congreso"dejó para otro tiempo" la decisión de la constitucionalidad. Cuatro años después, en 2013, retomaría la discusión y decidiría que es el tiempo de tomar medidas más efectivas, esto en el caso Shelby County.

En Shelby, inicia indicando que la VRA: "employed extraordinary measures to address an extraordinary problem", sobre esta superlativa hipótesis se sostendrá el resto de la argumentación.

¿En qué consiste esta medida extraordinaria? Hay dos elementos a tomar en cuenta y que responden a una discusión, que ha tomado décadas en la democracia americana.

Primero, la suposición de que bajo las enmiendas 14 y 15, se le otorgan "amplios poderes" al Congreso, para intervenir la soberanía estatal.

Segundo, estos amplios poderes, permiten al Congreso indicar que determinados estados de la Unión, tendrán la obligación de solicitar

${ }^{20}$ The Editors, “The Battle, not the War, on Voting Rights", The New York Times, 22 de junio de 2009. http://roomfordebate.blogs.nytimes.com/2009/06/22/the-battle-not-thewar-on-voting-rights/.

${ }^{21}$ The American Supreme Court, USA, The University of Chicago Press, 2010, p. 178. Algunos hacen una lectura sistemática de las decisiones tomadas bajo la presidencia de Roberts, en especial, los asuntos resueltos por él directamente, y la catalogan como una Corte que con paciencia, propone cambios en el diseño constitucional de los EUA: "The chief justice is a patient man playing a long game. He was content to wait four years to strike down a key provision of the Voting Rights Act. Apparently he likes to say I told you so." Richard L. Hasen, “The Chief Justice's long game”, The New York Times, 25 de junio de 2013. http://www.realclearpolitics.com/2013/06/26/the_chief_justice039s_long_ game_310447.html. 
Esta revista forma parte del acervo de la Biblioteca Jurídica Virtual del Instituto de Investigaciones Jurídicas de la UNAM

una "preautorización", para poder hacer modificaciones a su legislación electoral. Esto último implica dos cosas, la indicación de cuáles serán los estados — criterio de cobertura- y en qué consiste y cómo se solicita esta preautorización - criterio de procedencia- ${ }^{22}$

El criterio de cobertura está indicado en la sección 4 de la VRA, como hemos visto, mientras que el criterio procedimental en la sección 5.

Ahora, esta medida es extraordinaria porque al final, implica una afectación al diseño federal de los Estados Unidos, creando un criterio asimétrico de respeto - en cuanto a la legislación electoral estatal- y de reconocimiento de la soberanía local.

We explained that $₫ 5$ "imposes substantial federalism costs" and "differentiates between the States, despite our historic tradition that all the States enjoy equal sovereignty". 23

Al final, la sentencia se asienta sobre la respuesta a una pregunta, ¿aún sobrevive el problema extraordinario? El juez Roberts respondería que no, invalidando sólo la sección 4 de la ley, dejando sobrevivir la 5.

"The Act imposes current burdens and must be justified by current needs". 24

En este sentido, los aspectos que se deben resaltar de la decisión son cuatro:

1) Las reglas de la VRA que estaban en análisis son inusuales, fuera del marco constitucional normal, lo que el presidente Roberts calificó como: "strong medicine", ${ }^{25}$ pero, que se justificaban por las circunstancias extraordinarias de estados que históricamente

22 El juez Clarence Tomas expresaría en su voto particular, refiriéndose a la decisión en Austin, pero haciendo énfasis en la incisión federalista: “Though $\$ 5$ ’s preclearance requirement represented a "shar[p] depart[ure]" from "basic principles" of federalism and the equal sovereignty of the States" Shelby County v. Holder, 570 U.S. 2013 Concurring, p. 1. En adelante Shelby County (Con).

${ }^{23}$ Shelby County (Op), p. 7

${ }^{24}$ Ibidem, p. 9

25 Not only do States retain sovereignty under the Consti- tution, there is also a "fundamental principle of equal sovereignty" among the States... The Voting Rights Act sharply departs from these basic principles. It suspends "all changes to state election law - however innocuous - until they have been precleared by federal authorities in Washington, D. C.” Ibid., p. 10-11. 
Esta revista forma parte del acervo de la Biblioteca Jurídica Virtual del Instituto de Investigaciones Jurídicas de la UNAM

tenían un récord de irrespeto del derecho a los afroamericanos - como recordamos, este récord provenía de la guerra civil, en especial los estados del Sur-.

In 1966, we found these departures from the basic features of our system of government justified. The "blight of racial discrimination in voting" had "infected the electoral process in parts of our country for nearly a century." Katzenbach, 383 U. S., at 308. Several States had enacted a variety of requirements and tests "specifically designed to prevent" African-Americans from voting. ${ }^{26}$

2) La situación histórica del sur "Jim Crow", 27 justificaba la existencia del marco inusual derivado de las secciones 4 y 5 de la VRA. La dimensión histórica y la expectación implícita de la mejoría de la situación, proveniente de la política afirmativa de la VRA, hacía considerar que las medidas serían temporales, originalmente sólo contemplaban cinco años, pero como hemos visto, fue reiteradamente ratificada hasta 2006, cuando el Congreso reautorizó la medida por otros 25 años.

3) Las circunstancias históricas extraordinarias que justificaban la medida, ya han desaparecido, no hay evidencia del desequilibrio sistemático del goce del derecho de voto entre blancos y negros: "Nearly 50 years later, things have changed dramatically". ${ }^{28}$

4) La discriminación racial en el voto no ha sido totalmente eliminada, pero es dudable que siga existiendo en una extensión tan clara, como para seguir sosteniendo la irrupción en el marco

26 Ibidem, p. 13

${ }^{27}$ Lani Guiner, nos explica las implicaciones de esto: "Jim Crow-era system designed to make it more difficult for black people to register; relocating polling places so that blacks and Latinos would have to travel to remote or hostile venues in order to vote; cancelling elections or abolishing elected bodies at the moment that black or Latino candidates were on the verge of gaining a majority of seats on a governing body; using quotas in conducting annexations of unincorporated areas to prevent blacks from becoming a majority of the voting population; requiring Latino voters to attend citizenship hearings when their right to vote was challenged because they had a Spanish surname; and threatening students at a historically black college with prosecution if they registered to vote.", en "No Affirmative right to vote", Room for Debate, The Battle, Not the War, on Voting Rights, The New York Times, 22 de junio de 2009. http://roomfordebate.blogs.nytimes. com/2009/06/22/the-battle-not-the-war-on-voting-rights/.

28 Shelby County (Op), p. 14. 
Esta revista forma parte del acervo de la Biblioteca Jurídica Virtual del Instituto de Investigaciones Jurídicas de la UNAM

constitucional, afectación al federalismo y a la soberanía estatal. Refiriéndose a la información en la que se basó la emergencia de la VRA en 1965 y justificó la invasión a la soberanía estatal:

Respondents also rely heavily on data from the record compiled by Congress before reauthorizing the Act. Regardless of how one looks at that record, no one can fairly say that it shows anything approaching the "pervasive," "flagrant," "widespread," and "rampant" discrimination that clearly distinguished the covered jurisdictions from the rest of the Nation in $1965 .^{29}$

Por las anteriores razones, la Corte determinó que debe restaurar el orden constitucional. Esto quiere decir, que puede existir la pre-autorización, pero la fórmula para determinar Estados debe ser actualizada.

Lo anterior no quiere sostener la existencia de un estado de desprotección, sólo la eliminación de la situación extraordinaria. La VRA aún tiene medios para salvaguardar el derecho de voto, la sección 2 sigue vigente:

The VRA provides two major mechanisms to combat racial discrimination in voting: "vote dilution" claims under section 2 and the "preclearance requirement" under section 5 . Vote dilution claims under section 2 apply nationwide and create a private right of action for citizens and authorizes the Department of Justice to enforce the VRA's basic principle: " $[\mathrm{n}] \mathrm{o}$ voting qualification or prerequisite to voting or standard, practice, or procedure shall be imposed or applied by any State or political subdivision in a manner which results in a denial or abridgement of the right of any citizen of the United States to vote on account of race or color. ${ }^{30}$

Como vemos, estas disposiciones de la sección dos, permiten el litigio individual en caso de violación del derecho, el problema es -como dirían algunos - que resulta oneroso, tardado y la carga de la prueba está del lado de quien solicita el juicio. ${ }^{31}$

29 Shelby County (Sil), p. 3.

${ }^{30}$ Randall T. Adams, "Recent Development: Northwest Austin Municipal Utility District Number One v. Holder", Harvard Civil Rights-Civil Liberties Law Review, USA, 2010, vol. 45, núm. 1., Winter, p. 136.

31 "The decision allows those affected by voting rule changes to sue under Section 2 of the act, but that is a longer and more expensive process that places the burden of proof 
Esta revista forma parte del acervo de la Biblioteca Jurídica Virtual del Instituto de Investigaciones Jurídicas de la UNAM

La decisión tuvo dos votos más, el primero de ellos fue el concurrente del juez Clarence Thomas, quien afirmaría que debía irse más allá de la decisión y determinar la inconstitucionalidad de la sección 5 igualmente, algo que ya había sostenido en Nortwest Austin.

As the Court aptly notes: " $[\mathrm{N}] \mathrm{o}$ one can fairly say that [the record] shows anything approaching the 'pervasive,' 'flagrant,' 'widespread, and 'rampant' discrimination that faced Congress in 1965, and that clearly distinguished the covered jurisdictions from the rest of the Nation at that time." Ante, at 21. Indeed, circumstances in the covered jurisdictions can no longer be characterized as "exceptional" or "unique." "The extensive pattern of discrimination that led the Court to previously uphold $\$ 5$ as enforcing the Fifteenth Amendment no longer exists." Northwest Austin, supra, at 226 (THOMAS, J., concurring in judgment in part and dissenting in part). Section 5 is, thus, unconstitutional. ${ }^{32}$

Sin embargo, el voto de la juez Ruth Bader Ginsburg, es el diametralmente opuesto. Ella no encuentra justificación para determinar la inconstitucionalidad de la sección 4.

Plantea una interrogante importante, esta decisión la debe tomar ¿la Corte o el Congreso? Y con esto, retoma un debate profundo sobre la justificación democrática del Congreso para hacer esta ley, y la necesidad de una acción de igual naturaleza, para derogarla:

The question this case presents is who decides whether, as currently operative, $\$ 5$ remains justifiable, this Court, or a Congress charged with the obligation to enforce the post-Civil War Amendments "by appropriate legislation. ${ }^{33}$

En los casos de derecho de voto, señalaría la juez, la Corte debe diferir al Congreso, a quien se le dieron amplios poderes desde la Constitu-

on those challenging the changes." John Schwartz, "Shelvy County v. Holder. Between the Lines of the Voting Rights Act Opinion", The New York Times, 25 de junio de 2013. http://www.nytimes.com/interactive/2013/06/25/us/annotated-supreme-court-decisionon-voting-rights-act.html.

32 Shelby County (Con), p. 3.

${ }_{33}$ Shelby County v. Holder, 570 U.S. 2013 Dissenting, p. 1. En adelante Shelby County (Dis). 
Esta revista forma parte del acervo de la Biblioteca Jurídica Virtual del Instituto de Investigaciones Jurídicas de la UNAM

ción, y en especial devenidos de las enmiendas posteriores a la Guerra Civil, para proteger esos derechos. ${ }^{34}$

Para la juez, la abrumadora aprobación en ambas cámaras del Congreso, concluyeron por dos primordiales razones que la sección 5 debía continuar su validez: primero, la continuidad facilitará cumplimiento de los avances realizados; segundo, la continuidad servirá contra los posibles retrocesos.

The struggle for fairness in elections, is no over, though the tactics of those who would suppress voting have change. The court errs egregiously by overrriding Congress's decision.

Sobre la falta de necesidad de la medida, la juez afirma que si bien, las formas de afectación del derecho que se consideraron históricamente han sido ampliamente superadas, nuevas tácticas de represión del derecho han aparecido, llamadas "second-generation barriers":

Second-generation barriers come in various forms. One of the blockages is racial gerrymandering, the redrawing of legislative districts in an "effort to segregate the races for purposes of voting." Id., at 642. Another is adoption of a system of at-large voting in lieu of district-by-district voting in a city with a sizable black minority. By switch ing to at-large voting, the overall majority could control the election of each city council member, effectively elimi nating the potency of the minority's votes. ${ }^{35}$

En ese sentido, afirmaría la juez, la VRA es una legislación extraordinaria: "It is extraordinary because Congress embarked on a mission long delayed and of extraordinary importance: to realize the purpose and promise of the Fifteenth Amendment", ${ }^{36}$ en ese sentido, la Corte no debía suplantar una decisión tomada por el Congreso.

34 “The stated purpose of the Civil War Amendments was to arm Congress with the power and authority to protect all persons within the Nation from violations of their rights by the States", en Schwartz, John, op. cit.

35 Shelby County (Dis), p. 5.

36 Ibidem, p. 36. 


\section{Lecturas sobre su actualidad}

La actualidad y necesidad de la medida, sigue siendo discutida, porque como dice la juez Ginsburg, las restricciones históricas pueden haber sido superadas, pero algunos expertos plantean nuevas amenazas, diferentes grupos sociales afectados, que se anexan a la cuestión racial que originalmente ha combatido.

¿Es la ley todavía necesaria? En la elección presidencial de 2012, se observó que oficiales estatales y locales, intentaron establecer medidas para evadir el cumplimiento del derecho de voto de manera universal Por ejemplo, la implementación de leyes para restringir documentación de identidad para votar, acortar las horas para votar temprano, hacer más difícil el registro de voto. Estas decisiones tendrían impacto en la juventud, la tercera edad y aquellos calificados dentro de la brecha de pobreza.

These efforts included Texas's stringent voter ID law, which would have disenfranchised low-income Texas citizens, who are disproportionately African-American and Hispanic, as well as the state's discriminatory redistricting plan, and Florida's attempts to slash the period for early voting, which is disproportionally used by African-American voters. ${ }^{37}$

Otra de las circunstancias que quedan pendientes es el tema de los latinos, el profesor Rodolfo de la Garza, da una serie de circunstancias que pueden señalar la necesidad de la medida: "Community organizers report that many Latino citizens stay home rather than risk being bullied, politically hounded or arrested if they try to vote. Latino history offers so many examples of such treatment that it is probably a major factor explaining why Latino voter turnout is 10 percent lower than Anglo's". 38

37 Wydra, Elizabeth, "2012 Showed Us the Law is Still Needed", Room for Debate, The New York Times, 24 de febrero de 2013, http://www.nytimes.com/roomfordebate/2013/02/2 4/ is-the-voting-rights-act-still-needed/how-2012-shows-the-voting-rights-act-is-still-needed.

38 "A voice for latino voters", cit., http://www.nytimes.com/roomfordebate/2013/02/24/ is-the-voting-rights-act-still-needed/the-voting-rights-act-protects-latino-voters. 
Esta revista forma parte del acervo de la Biblioteca Jurídica Virtual del Instituto de Investigaciones Jurídicas de la UNAM

Desafortunadamente, diría el profesor de la Garza, la lucha de los latinos por su participación democrática, no ha sido tan reconocida como la lucha por los derechos civiles.

Otra dimensión faltante, la juventud se convierte en una fuerza electoral poderosa, cerca de 11500 americanos llegan a los 18 años cada día, nos dice la profesor Heather Smith, y enfrentan retos en su derecho a votar, como la potencial negación de los estudiantes a votar en el lugar donde viven y van a clases:

Florida passed a law that restricted volunteer-led voter registration drives on college campuses and in communities. This law was blocked, also for its violation of the U.S. Constitution. And numerous states passed laws with strict voter identification requirements that disproportionately affected young voters. For example, in Texas, a new law allowed for concealedgun licenses as proof of identification at the polls, but not a student identification card from a state college or university. This voter identification provision was blocked as discriminatory under the Voting Rights Act. ${ }^{39}$

Quizás un alivio a esta medida, que algunos pueden denominar desproporcionada, de eliminar una de las disposiciones más efectivas para combatir la discriminación política en los Estados Unidos, es la afirmación que la misma Corte, hiciera en este mismo término, en el caso Arizona vs. Interval Council of Arizona, ${ }^{40}$ donde el juez Scalia escribiendo el voto mayoritario- determinaría inconstitucional, una ley que obliga a cualquiera que quisiera votar, a otorgar prueba de su ciudadanía - por supuesto hay que tener presente que este tipo de leyes en estados como Arizona, están animadas por el tema migratorio-. La decisión reafirma la disposición federal conocida como motor voter, ${ }^{41}$ que sólo requiere una declaración jurada.

39 "The Law protect joung voters", cit., http://www.nytimes.com/roomfordebate/2013/02 /24/is-the-voting-rights-act-still-needed/the-voting-rights-act-protects-young-voters.

${ }_{40}$ Resuelta el 17 de junio de 2013, con una votación 7 a 2. http://www.scotusblog.com/ case-files/cases/arizona-v-the-inter-tribal-council-of-arizona-inc/.

41 "requires states to "accept and use" a national voter registration form for federal elections. Voters using the form have to swear, under penalty of perjury, that they are citizens, but they do not have to provide a driver's license, passport or other proof of citizenship", Editorial Board, “The Court: Congress regulates federal elections”, The New York Times, 17 de junio de 2013, http://www.nytimes.com/2013/06/18/opinion/the-courtcongress-regulates-federal-elections.html. 
Esta revista forma parte del acervo de la Biblioteca Jurídica Virtual del Instituto de Investigaciones Jurídicas de la UNAM

The elections clause, by contrast, does not speak to racial discrimination at all, but addresses the administration of voting rules. Still, in light of the Supreme Court's ruling in Shelby County, it could have an important role to play. ${ }^{42}$

Por último, debemos retomar que el problema en Shelby, también es sobre el diseño federal de los Estados Unidos. La Corte efectivamente aún deja abierta la posibilidad de actualizar la fórmula de la sección 4 , y con esto legitima de alguna manera la acción de Congreso al momento de legislar la VRA bajo el mandato de la Enmienda 15, en este sentido, parece que está refrendando las facultades arrogadas por el Congreso. Es posible, por tanto, una nueva determinación, pero debe reflejar una situación extraordinaria, para sostener una medida igualmente extraordinaria dentro del diseño constitucional de los Estados Unidos, una decisión que necesitaría un acuerdo, que se sabe, es difícil de volver a alcanzar al interior del Congreso.

${ }^{42}$ Wegman, Jesse, "Plan B for voting rights", The New York Times, 31 de agosto de 2013, http://www.nytimes.com/2013/09/01/opinion/sunday/plan-b-for-voting-rights.html?_r=0. 